\title{
The Ingestion of High-Fructose Syrup-Containing Cola with a Hamburger Delays Postprandial Lipid Metabolism in Young Healthy Japanese Women
}

\author{
Hiromi Saito $^{1,2}$, Maiko Kato ${ }^{1}$, Akihiro Yoshida $^{3}$, Michitaka Naito ${ }^{1}$ \\ ${ }^{1}$ Division of Nutrition \& Health, School \& Graduate School of Life Studies, Sugiyama Jogakuen University, Nagoya, Japan \\ ${ }^{2}$ Department of Food Science and Nutrition, Nara Women's University, Nara, Japan \\ ${ }^{3}$ Department of Clinical Laboratory, Nakatsugawa Municipal General Hospital, Nakatsugawa, Japan \\ Email address: \\ naito@sugiyama-u.ac.jp (M. Naito)
}

\section{To cite this article:}

Hiromi Saito, Maiko Kato, Akihiro Yoshida, Michitaka Naito. The Ingestion of High-Fructose Syrup-Containing Cola with a Hamburger Delays Postprandial Lipid Metabolism in Young Healthy Japanese Women. Journal of Food and Nutrition Sciences.

Vol. 3, No. 4, 2015, pp. 139-146. doi: 10.11648/j.jfns.20150304.11

\begin{abstract}
Aim: To investigate the acute effects of the ingestion of high-fructose syrup (HFS)-containing cola in combination with a hamburger on postprandial lipid metabolism. Methods: Twelve young healthy women with apolipoprotein E phenotype $3 / 3$ were enrolled in the study. Each subject underwent 3 test trials in a randomized crossover design. The trials were as follows: $\mathrm{C}$ trial, cola (350 mL, containing HFS); H trial, 1 hamburger; $\mathrm{CH}$ trial, cola + hamburger. Venous blood samples were collected at fasting levels and at $0.5,1,2,4$, and $6 \mathrm{~h}$ after ingestion. Results: The serum concentrations of triglyceride (TG), remnant-like particle (RLP)-TG, and apolipoprotein B-48 (apoB48) peaked at $2 \mathrm{~h}$ in the $\mathrm{H}$ trial and at $4 \mathrm{~h}$ in the $\mathrm{CH}$ trial. Compared with each fasting level, the serum TG concentration in the $\mathrm{H}$ trial was significantly increased at 1,2 , and $4 \mathrm{~h}$, and it returned to the fasting level at $6 \mathrm{~h}$. However, compared with each fasting level, the TG concentration in the $\mathrm{CH}$ trial was significantly increased at 4 and $6 \mathrm{~h}$, and it did not return to the fasting level at $6 \mathrm{~h}$. The serum apoB48 concentration peaked at $2 \mathrm{~h}$ in the $\mathrm{H}$ trial and at $4 \mathrm{~h}$ in the $\mathrm{CH}$ trial. The apoB48 concentration at 4 and $6 \mathrm{~h}$ and the incremental area under the curve for apoB48 in the $\mathrm{CH}$ trial tended to be higher than those in the $\mathrm{H}$ trial. Conclusion: The ingestion of cola in combination with a hamburger delayed and extended postprandial lipidemia in comparison with the ingestion of a hamburger only, suggesting that fructose contained in the cola exacerbates postprandial lipid metabolism.
\end{abstract}

Keywords: Fructose, Apolipoprotein B-48, Postprandial Lipidemia, Triglyceride-Rich Lipoprotein, Fast Food

\section{Introduction}

The production and use of high-fructose syrup (HFS) for soft drinks has been increasing both in the USA and in Japan $[1,2]$. Overconsumption of sugar-sweetened beverages has been related to obesity in children and adults [3, 4]. A prospective study in middle-aged Japanese using a self-administered food-frequency questionnaire indicated that soft drink intake is positively associated with the risk of total stroke and, more specifically, ischemic stroke for women [5]. In young men and women, after 2-week consumption of an energy-balanced diet containing a 25\%-energy, sugar-sweetened (with fructose or HFS, but not glucose) beverage, the area under the curve (AUC) for 24-h serum triglyceride (TG) increased [6]. In addition, increased postprandial triglyceride-rich lipoprotein (TRL) has been reported to be a major risk factor for coronary heart disease [7].

In our previous studies, postprandial $\mathrm{TG}$, remnant-like particle (RLP)-TG, and apolipoprotein B-48 (apoB48) concentrations following the ingestion of fructose $(0.5 \mathrm{~g} / \mathrm{kg}$ body mass $)$ in combination with fat cream $(0.35 \mathrm{~g} / \mathrm{kg}$ as fat $)$ were found to be significantly higher than those following the ingestion of glucose [8]. The ingestion of an HFS-containing beverage in combination with fat cream exacerbated both exogenous and endogenous lipid metabolism, and the ratio of fructose to glucose contained in the beverage was a key factor in the metabolic disturbance when the sugar load was equicaloric [9].

In the present study, we investigated postprandial 
lipoprotein metabolism indices following the ingestion of daily used fast food in young female students. This group was chosen because the ingestion of fast food has been reported to be higher in college students than in high school- or elementary school-aged pupils in Japan [10]. Cola and hamburgers are representative examples of fast food; cola contains a high amount of HFS, and a hamburger contains a high amount of fat, particularly saturated fatty acids.

The aim of the present study was to investigate the acute effects of the ingestion of an HFS-containing beverage (cola) in combination with a hamburger on postprandial exogenous and endogenous lipid metabolism in healthy young Japanese women.

\section{Methods}

\subsection{Subjects}

Twelve young healthy Japanese women with normal ovarian cycles and apolipoprotein E (apoE) phenotype 3/3 were enrolled as participants. All the subjects were nonsmokers, had no apparent acute or chronic illnesses, and were not taking any medications or dietary supplements. This study was approved by the Institutional Review Board of the Sugiyama Jogakuen University School of Life Studies (No. 2013-2), and each subject provided written informed consent for study participation.

\subsection{Anthropometric and Body Composition Measurement}

Body mass and height were measured according to standard methods. Waist circumference was assessed as the abdominal girth at the level of the umbilicus, and hip circumference was measured at the level of the greater trochanters. The waist-to-hip $(\mathrm{W} / \mathrm{H})$ ratio was calculated. Body composition, including the visceral fat area (VFA), was analyzed using an eight-polar bioelectrical impedance method with the InBody720 (Biospace, Tokyo, Japan).

\subsection{Cola and Hamburger Load Test}

Each subject underwent 3 test trials in a randomized crossover design. The trials were as follows: $\mathrm{C}$ trial, cola (Coca-Cola, $350 \mathrm{~mL}$, Coca-Cola Journey, Tokyo, Japan); H trial, 1 hamburger (Cheeseburger, $122 \mathrm{~g}$, McDonald's, Tokyo, Japan); $\mathrm{CH}$ trial, cola + hamburger. The composition of cola and hamburger were analyzed at Falco Life Science (Kyoto, Japan) (Tables 1 \& 2).

Table 1. Composition of hamburger.

\begin{tabular}{ll}
\hline Energy (kcal) & 314 \\
Carbohydrate $(\mathrm{g})$ & 32.5 \\
Protein $(\mathrm{g})$ & 15.9 \\
Fat $(\mathrm{g})$ & 12.7 \\
Saturated fatty acid $(\mathrm{g})$ & 7.3 \\
Monounsaturated fatty acid (g) & 4.7 \\
Polyunsaturated fatty acid (g) & 0.8 \\
Cholesterol (mg) & 44 \\
Dietary fiber (g) & 3.1 \\
\hline
\end{tabular}

Values of 1 hamburger (122 g) are shown.
Table 2. Composition of cola.

\begin{tabular}{ll}
\hline Energy (kcal) & 158 \\
Carbohydrate (g) & 37.5 \\
Fructose (g) & 16.8 \\
Glucose (g) & 12.6 \\
Sucrose (g) & 8.1 \\
Protein (g) & 0.0 \\
Fat (g) & 0.0 \\
\hline
\end{tabular}

Values per $350 \mathrm{~mL}$ of cola are shown.

\subsection{Experimental Design}

Subjects abstained from consuming caffeine or alcohol during the day before the experiment, and ingested cola and/or hamburger after a 12-h overnight fast. Venous blood samples were obtained before ingestion $(0 \mathrm{~h})$ and at $0.5,1,2,4$, and $6 \mathrm{~h}$ after ingestion. During the test, subjects avoided exercise and eating but had free access to water after $1 \mathrm{~h}$. All blood samples were collected while subjects were in a supine position. There was a 4-week interval between the test days to minimize the confounding effects of the menstrual status on lipid metabolism.

\subsection{Biochemical Analysis}

The serum samples were immediately refrigerated at $4^{\circ} \mathrm{C}$ or frozen at $-80^{\circ} \mathrm{C}$ until analysis. The level of fructose was enzymatically measured (BioAssay Systems, CA, USA). The level of glucose was measured using a mutarotase-glucose oxidase method (Wako, Osaka, Japan). The level of insulin was measured using a chemiluminescent enzyme immunoassay (Fujirebio, Tokyo, Japan). Insulin resistance was evaluated according to the homeostasis model assessment for insulin resistance (HOMA-IR) [11]. The hemoglobin A1c (HbA1c) level was measured using a latex agglutination method (Fujirebio) and expressed as the National Glycohemoglobin Standardization Program (NGSP) value. The levels of free fatty acids (FFA) (Eiken Chemical, Tokyo, Japan) and lactate (Kyowa Medex, Tokyo, Japan) were measured enzymatically. The level of total cholesterol (TC) was measured enzymatically (Sysmex, Hyogo, Japan). The level of high-density lipoprotein-cholesterol (HDL-C) was measured using a direct method (Fujirebio), while the level of low-density lipoprotein-cholesterol (LDL-C) was calculated using the Friedewald formula. The level of TG was enzymatically measured (Sekisui Medical, Tokyo, Japan). The TG level in the TG-rich lipoproteins was estimated using a surfactant-based homogeneous assay (VLDL-TG ${ }^{\text {TM }}$, Shino-test, Tokyo, Japan) [12] and shown as hepatic TRL-triglyceride (hTRL-TG), as described previously [9]. This assay determines $100 \%$ of TG in very-low-density lipoprotein (VLDL) and $79.0 \%$ in intermediate density lipoprotein (IDL); however, it also determines $16.1 \%$ of TG in chylomicron (CM) and $29.5 \%$ in LDL [12]. The level of RLP-TG was measured using an immunosorbent assay (Otsuka Pharmaceutical, Tokyo, Japan), and the level of remnant lipoprotein-cholesterol (RemL-C) using a 
homogeneous assay (MetaboRead ${ }^{\mathrm{TM}}$, Kyowa Medex, Japan). The level of apolipoproteins (apo) AI, AII, B(-100), CII, CIII, and $\mathrm{E}$ were measured using an immunoturbidimetric method (Sekisui Medical). The level of apoB48 was measured by chemiluminescent enzyme immunoassay (Fujirebio). The apoE phenotype was measured using the isometric electrophoresis method (Phenotyping ApoE IEF System ${ }^{\mathrm{TM}}$, Joko, Tokyo, Japan).

\subsection{Quantification of Postprandial Metabolism}

Postprandial changes in the concentrations of $\mathrm{TG}$, hTRL-TG, RLP-TG, RemL-C, apoB and apoB48 were calculated as the difference from the baseline mean value (as 0 at $0 \mathrm{~h}$ ) and shown as $\Delta \mathrm{TG}, \Delta \mathrm{hTRL}-\mathrm{TG}, \Delta \mathrm{RLP}-\mathrm{TG}, \Delta \mathrm{RemL}-\mathrm{C}$, $\triangle \mathrm{apoB}$, and $\Delta \mathrm{apoB} 48$, respectively. The postprandial metabolism was quantified by calculating the incremental area under the curve ( $\triangle \mathrm{AUC})$, which was defined as the difference between AUC and the area below baseline $(0 \mathrm{~h})$ from 0 to $6 \mathrm{~h}$, as described previously [9].

\subsection{Statistics}

All data were expressed as mean \pm SEM. The statistical analyses were performed using SPSS ver. 19 (IBM, Tokyo, Japan). Differences in the time course compared with the fasting values were analyzed using the Friedman test, followed by the Wilcoxon signed-rank test with the Bonferroni correction. The differences in the values at each time point in the 3 trials were assessed using the Kruskal-Wallis test, followed by the Mann-Whitney U-test with the Bonferroni correction.

\section{Results}

\subsection{Physical Characteristics and Fasting Blood Data}

The physical characteristics and fasting blood chemistry data of the subjects are shown in Table 3. There were no significant differences in any of the physical characteristics among the 3 trials.

Table 3. Anthropometric and clinical characteristics.

\begin{tabular}{|c|c|c|c|c|c|c|c|c|c|}
\hline Trial & \multicolumn{3}{|c|}{$\mathrm{C}$} & \multicolumn{3}{|c|}{$\mathbf{H}$} & \multicolumn{3}{|c|}{$\mathrm{CH}$} \\
\hline Age (years) & 20.3 & \pm & 0.2 & & & & & & \\
\hline Height $(\mathrm{cm})$ & 157.2 & \pm & 1.3 & & & & & & \\
\hline Weight (kg) & 47.9 & \pm & 1.5 & 47.8 & \pm & 1.3 & 47.7 & \pm & 1.5 \\
\hline BMI $\left(\mathrm{kg} / \mathrm{m}^{2}\right)$ & 19.4 & \pm & 0.5 & 19.4 & \pm & 0.4 & 19.4 & \pm & 0.5 \\
\hline Waist (cm) & 67.4 & \pm & 2.0 & 67.1 & \pm & 1.7 & 66.2 & \pm & 1.6 \\
\hline $\mathrm{W} / \mathrm{H}$ & 0.78 & \pm & 0.01 & 0.78 & \pm & 0.02 & 0.77 & \pm & 0.01 \\
\hline $\operatorname{VFA}\left(\mathrm{cm}^{2}\right)$ & 22.2 & \pm & 4.0 & 22.9 & \pm & 3.6 & 20.7 & \pm & 3.7 \\
\hline HbAlc (\%) & 5.3 & \pm & 0.1 & 5.3 & \pm & 0.1 & 5.3 & \pm & 0.1 \\
\hline HOMA-IR & 1.1 & \pm & 0.1 & 1.2 & \pm & 0.2 & 1.1 & \pm & 0.1 \\
\hline $\mathrm{TC}(\mathrm{mg} / \mathrm{dL})$ & 157.1 & \pm & 6.9 & 160.0 & \pm & 6.6 & 161.3 & \pm & 5.7 \\
\hline LDL-C (mg/dL) & 84.5 & \pm & 5.3 & 84.3 & \pm & 4.6 & 87.6 & \pm & 4.8 \\
\hline HDL-C (mg/dL) & 62.3 & \pm & 3.2 & 65.0 & \pm & 3.7 & 63.8 & \pm & 3.2 \\
\hline apoAI (mg/dL) & 140.3 & \pm & 4.0 & 145.9 & \pm & 5.6 & 142.3 & \pm & 4.9 \\
\hline apoAII (mg/dL) & 24.8 & \pm & 0.4 & 26.1 & \pm & 0.5 & 25.6 & \pm & 0.6 \\
\hline apoCIII (mg/dL) & 6.2 & \pm & 0.5 & 6.8 & \pm & 0.5 & 6.7 & \pm & 0.4 \\
\hline apoE (mg/dL) & 3.7 & \pm & 0.2 & 3.8 & \pm & 0.1 & 3.9 & \pm & 0.1 \\
\hline $\mathrm{SBP}(\mathrm{mmHg})$ & 102.7 & \pm & 2.5 & 102.7 & \pm & 2.6 & 103.6 & \pm & 3.0 \\
\hline DBP (mmHg) & 67.4 & \pm & 1.8 & 66.6 & \pm & 1.7 & 65.0 & \pm & 1.5 \\
\hline PR (beats/min) & 72.0 & \pm & 2.0 & 74.3 & \pm & 2.4 & 73.0 & \pm & 1.9 \\
\hline
\end{tabular}

All values are presented as mean \pm SEM.

SBP: systolic blood pressure, DBP: diastolic blood pressure, PR: pulse rate

\subsection{Serum Concentrations of Fasting and Postprandial Fructose, Glucose, Insulin, FFA, and Lactate}

The serum concentrations of fasting and postprandial fructose, glucose, insulin, FFA, and lactate in the 3 trials are presented in Table 4.

The serum concentration of fructose was significantly increased at $0.5,1$, and $2 \mathrm{~h}$ in the $\mathrm{C}$ and $\mathrm{CH}$ trials compared with each fasting value ( $p<0.05$ for all) and then returned to each fasting value at $4 \mathrm{~h}$. The serum fructose concentration in the $\mathrm{H}$ trial showed no significant change during the $6 \mathrm{~h}$.

The serum concentration of glucose was significantly increased at $0.5 \mathrm{~h}$ in the $\mathrm{C}$ trial and at 0.5 and $1 \mathrm{~h}$ in the $\mathrm{H}$ and
$\mathrm{CH}$ trials compared with each fasting value ( $p<0.05$ for all). At $0.5 \mathrm{~h}$, the serum glucose concentration in the $\mathrm{C}$ and $\mathrm{CH}$ trials was significantly higher than that in the $\mathrm{H}$ trial $(p<0.05$ for all).

The serum concentration of insulin in the 3 trials peaked at $0.5 \mathrm{~h}$ and returned to (or below) fasting levels at $2 \mathrm{~h}$ in the $\mathrm{C}$ trial and at $4 \mathrm{~h}$ in the $\mathrm{H}$ and $\mathrm{CH}$ trials. The serum concentration of insulin in the $\mathrm{CH}$ trial was higher at 1 and $2 \mathrm{~h}$ than that in the $\mathrm{C}$ trial and at 0.5 and $1 \mathrm{~h}$ than that in the $\mathrm{H}$ trial $(p<0.05$ each). At $1 \mathrm{~h}$, it was also higher in the $\mathrm{H}$ trial than in the $\mathrm{C}$ trial $(p<0.05)$.

The serum concentration of FFA instantly decreased in the 3 trials following ingestion, reaching its lowest level at $1 \mathrm{~h}$ in the 
$\mathrm{C}$ trial, and at $2 \mathrm{~h}$ in the $\mathrm{H}$ and $\mathrm{CH}$ trials, followed by an increase in all trials. The serum concentration of FFA in the $\mathrm{CH}$ trial was significantly lower at 1 and $2 \mathrm{~h}$ than in the $\mathrm{H}$ trial and at 2 and $4 \mathrm{~h}$ than in the $\mathrm{C}$ trial $(p<0.05$ for all). At $4 \mathrm{~h}$, it was also lower in the $\mathrm{H}$ trial than in the $\mathrm{C}$ trial $(p<0.05)$.

The serum lactate concentration in the 3 trials was significantly increased at $1 \mathrm{~h}$ compared with each fasting value ( $p<0.05$ for all) and then returned to the fasting levels at $2 \mathrm{~h}$ in the $\mathrm{H}$ and $\mathrm{CH}$ trials and at $4 \mathrm{~h}$ in the $\mathrm{C}$ trial. The serum lactate concentration in the $\mathrm{C}$ and $\mathrm{CH}$ trials was significantly higher than that in the $\mathrm{H}$ trial at $1 \mathrm{~h}$ ( $p<0.05$ for both).

Table 4. The values of fasting and postprandial fructose, glucose, insulin, FFA, and lactate.

\begin{tabular}{|c|c|c|c|c|c|c|}
\hline Time (h) & $\mathbf{0}$ & 0.5 & 1 & 2 & 4 & 6 \\
\hline \multicolumn{7}{|c|}{ Fructose (mg/dL) } \\
\hline $\mathrm{C}$ & $0.65 \pm 0.04$ & $\left.5.65 \pm 0.46^{*}\right] \#$ & $\left.3.93 \pm 0.36^{*}\right]$ & $2.18 \pm 0.20 * 7 \#$ & $0.71 \pm 0.06$ & $0.66 \pm 0.05$ \\
\hline $\mathrm{H}$ & $0.59 \pm 0.04$ & $0.66 \pm 0.04$ & $0.76 \pm 0.04$ 」\# & $0.69 \pm 0.08$ & $0.59 \pm 0.05$ & $0.62 \pm 0.06$ \\
\hline $\mathrm{CH}$ & $0.67 \pm 0.05$ & $\left.5.65 \pm 0.46^{*}\right\rfloor \#$ & $3.76 \pm 0.38 *] \#$ & $2.08 \pm 0.20 *] \#$ & $0.81 \pm 0.10$ & $0.72 \pm 0.05$ \\
\hline \multicolumn{7}{|c|}{ Glucose (mg/dL) } \\
\hline $\mathrm{C}$ & $88.3 \pm 1.7$ & $133.6 \pm 3.2^{*}$ & $103.9 \pm 6.2$ & $91.7 \pm 2.7$ & $85.6 \pm 1.3$ & $87.4 \pm 1.2$ \\
\hline $\mathrm{H}$ & $86.4 \pm 1.8$ & $103.4 \pm 4.0 *\rfloor \#$ & $98.5 \pm 4.1^{*}$ & $90.7 \pm 4.2$ & $85.8 \pm 1.6$ & $82.6 \pm 1.5$ \\
\hline \multicolumn{7}{|c|}{ Insulin $(\mu \mathrm{U} / \mathrm{mL})$} \\
\hline $\mathrm{C}$ & $5.14 \pm 0.50$ & $44.10 \pm 4.95^{*}$ & $\left.18.21 \pm 2.50^{*}\right\urcorner \#$ & $8.39 \pm 1.08$ & $3.36 \pm 0.35^{*}$ & $3.51 \pm 0.26$ \\
\hline $\mathrm{H}$ & $5.50 \pm 0.75$ & $31.20 \pm 5.27 *$ & $29.88 \pm 2.58 * \frac{1+}{7+} \mid \#$ & $16.84 \pm 3.00 *$ & $3.54 \pm 0.49^{*}$ & $3.15 \pm 0.42 *$ \\
\hline $\mathrm{CH}$ & $4.76 \pm 0.60$ & $66.03 \pm 9.55 *\rfloor^{\#}$ & $\left.\left.46.78 \pm 5.16^{*}\right\rfloor \#\right\rfloor$ & $29.78 \pm 4.56 *]$ & $5.12 \pm 0.80$ & $3.12 \pm 0.37^{*}$ \\
\hline \multicolumn{7}{|c|}{$\mathrm{FFA}(\mu \mathrm{mol} / \mathrm{L})$} \\
\hline $\mathrm{C}$ & $571.1 \pm 63.6$ & & $138.4 \pm 28.4^{*}$ & $186.9 \pm 38.5^{*}$ & $828.8 \pm 76.2 * 7 \#$ & $967.8 \pm 68.6^{*}$ \\
\hline $\mathrm{H}$ & $522.0 \pm 70.1$ & & $220.5 \pm 27.6^{*}$ & $173.5 \pm 30.9 *$ & $526.0 \pm 49.7^{\perp \#}$ & $883.0 \pm 81.3^{*}$ \\
\hline \multicolumn{7}{|c|}{ Lactate $(\mathrm{mg} / \mathrm{dL})$} \\
\hline $\mathrm{C}$ & $9.2 \pm 0.5$ & & $\left.18.1 \pm 1.4^{*}\right\rceil \#$ & $13.0 \pm 0.9^{*}$ & $9.9 \pm 0.7$ & $10.5 \pm 1.0$ \\
\hline $\mathrm{H}$ & $10.1 \pm 1.0$ & & $11.7 \pm 0.9 *$ & $10.8 \pm 0.6$ & $8.2 \pm 1.1$ & $9.1 \pm 0.9$ \\
\hline $\mathrm{CH}$ & $9.9 \pm 1.3$ & & $\left.21.5 \pm 1.6^{*}\right]^{\#}$ & $13.2 \pm 0.9$ & $9.1 \pm 0.6$ & $9.6 \pm 1.1$ \\
\hline
\end{tabular}

All values are presented as mean \pm SEM. ${ }^{*} p<0.05$ compared with the fasting value in each trial. \#p $<0.05$ when compared among the trials.

\subsection{Serum Concentrations of Fasting and Postprandial TG, hTRL-TG, RLP-TG, and RemL-C}

The concentrations of fasting and postprandial $\mathrm{TG}$,
hTRL-TG, RLP-TG, and RemL-C in the 3 trials are presented in Table 5, and the time courses of $\Delta \mathrm{TG}, \triangle \mathrm{hTRL}-\mathrm{TG}$, $\triangle \mathrm{RLP}-\mathrm{TG}$, and $\Delta$ RemL-C are shown in Fig. $1 \mathrm{~A}-\mathrm{D}$.

Table 5. The values of fasting and postprandial TG, hTRL-TG, RLP-TG, RemL-C, apoB, and apoB48.

\begin{tabular}{|c|c|c|c|c|c|c|}
\hline Time (h) & $\mathbf{0}$ & 1 & 2 & 4 & 6 & \\
\hline \multicolumn{7}{|c|}{$\mathrm{TG}(\mathrm{mg} / \mathrm{dL})$} \\
\hline $\mathrm{C}$ & $51.3 \pm 6.9$ & $50.3 \pm 6.2$ & $44.4 \pm 6.3$ & $55.4 \pm 6.8$ & $56.3 \pm 6.4$ & \\
\hline $\mathrm{H}$ & $53.8 \pm 6.9$ & $62.3 \pm 7.6^{*}$ & $\left.75.6 \pm 10.1^{*}\right\rfloor^{\#}$ & $67.1 \pm 10.0 *$ & $59.6 \pm 7.8$ & \\
\hline $\mathrm{CH}$ & $50.1 \pm 4.9$ & $49.9 \pm 5.4$ & $56.0 \pm 6.5$ & $73.8 \pm 9.3^{*}$ & $63.3 \pm 9.7^{*}$ & \\
\hline \multicolumn{7}{|c|}{ hTRL-TG (mg/dL) } \\
\hline $\mathrm{C}$ & $41.4 \pm 6.7$ & $40.7 \pm 6.1$ & $35.9 \pm 6.3$ & $47.1 \pm 7.0$ & $48.8 \pm 6.5$ & \\
\hline $\mathrm{H}$ & $45.1 \pm 7.2$ & $51.4 \pm 7.5^{*}$ & $55.5 \pm 8.4^{*}$ & $52.7 \pm 9.3$ & $47.1 \pm 7.9$ & \\
\hline \multicolumn{7}{|c|}{ RLP-TG (mg/dL) } \\
\hline $\mathrm{C}$ & $8.3 \pm 1.0$ & $9.9 \pm 1.0$ & $7.2 \pm 1.0$ & $9.8 \pm 1.0$ & $10.6 \pm 1.0$ & \\
\hline $\mathrm{H}$ & $9.5 \pm 1.4$ & $16.3 \pm 2.7^{*}$ & $\left.25.0 \pm 3.6^{*}\right\rfloor \#$ & $\left.18.1 \pm 3.3^{*}\right\rfloor^{\#}$ & $13.8 \pm 2.0^{*}$ & \\
\hline $\mathrm{CH}$ & $8.3 \pm 0.9$ & $10.8 \pm 1.0^{*}$ & $16.8 \pm 2.0^{*}$ & $27.2 \pm 4.3 *$ & $14.3 \pm 1.2^{*}$ & \\
\hline \multicolumn{7}{|c|}{ RemL-C (mg/dL) } \\
\hline C & $3.6 \pm 0.6$ & $3.5 \pm 0.5$ & $3.2 \pm 0.5$ & $3.7 \pm 0.5$ & $3.9 \pm 0.4$ & \\
\hline $\mathrm{H}$ & $3.9 \pm 0.5$ & $4.2 \pm 0.5$ & $4.5 \pm 0.6^{*}$ & $4.6 \pm 0.7 *$ & $4.6 \pm 0.6^{*}$ & \\
\hline \multicolumn{7}{|c|}{ apoB (mg/dL) } \\
\hline $\mathrm{C}$ & $61.4 \pm 3.4$ & $61.3 \pm 3.4$ & $61.2 \pm 3.4$ & $62.8 \pm 3.5$ & $63.4 \pm 3.6^{*}$ & \\
\hline $\mathrm{H}$ & $61.5 \pm 3.2$ & $60.5 \pm 2.9$ & $59.1 \pm 2.9^{*}$ & $60.8 \pm 3.2$ & $62.8 \pm 3.1$ & \\
\hline $\mathrm{CH}$ & $62.7 \pm 3.1$ & $61.5 \pm 3.4$ & $60.9 \pm 3.0^{*}$ & $61.0 \pm 3.2 *$ & $63.6 \pm 3.5$ & \\
\hline \multicolumn{7}{|c|}{ apoB48 $(\mu \mathrm{g} / \mathrm{mL})$} \\
\hline $\mathrm{C}$ & $1.5 \pm 0.3$ & $1.7 \pm 0.2$ & $0.4 \pm 0.3 \quad\urcorner \#$ & $1.2 \pm 0.2$ & $1.6 \pm 0.2$ & \\
\hline $\mathrm{H}$ & $1.7 \pm 0.4$ & $\left.3.4 \pm 0.6^{*}\right\rfloor^{\#} \mid \#$ & $\left.3.7 \pm 0.4^{*}\right\rfloor^{\#}$ & $\left.3.3 \pm 0.4^{*}\right\rfloor^{\#}$ & $\left.2.8 \pm 0.4^{*}\right\lrcorner^{\#}$ & \# \\
\hline $\mathrm{CH}$ & $1.5 \pm 0.2$ & $2.6 \pm 0.2 *]$ & $3.5 \pm 0.3^{*}$ & $\left.4.3 \pm 0.5^{*}\right]$ & $3.3 \pm 0.3 *$ & ] \\
\hline
\end{tabular}

All values are presented as mean \pm SEM. $* p<0.05$ compared with the fasting value in each trial. 

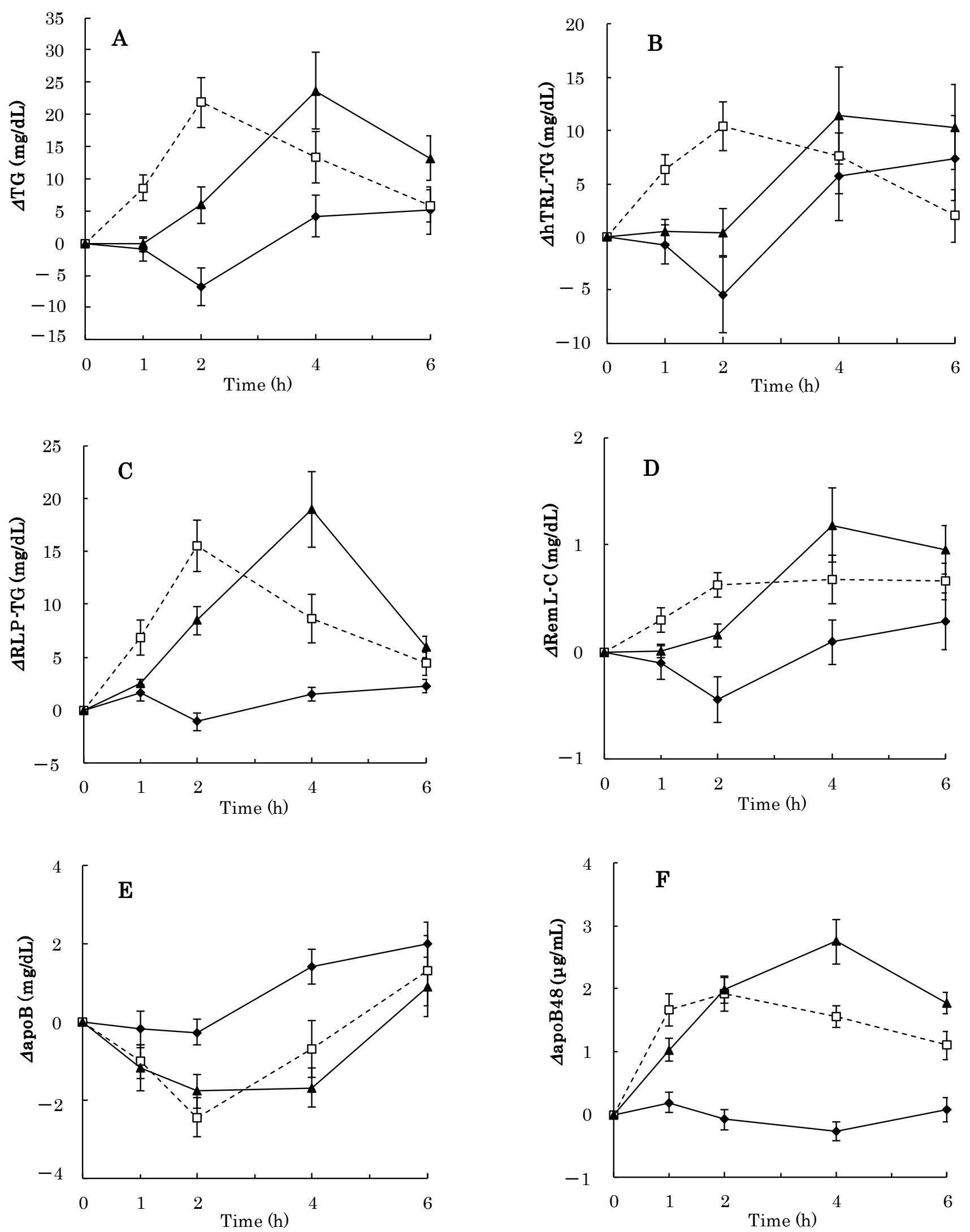

$$
\longrightarrow \mathrm{C} \quad--\mathrm{a}-\mathrm{H} \longrightarrow \mathrm{CH}
$$

Fig. 1. Postprandial values of $\triangle T G(A), \triangle h T R L-T G(B), \triangle R L P-T G(C), \triangle R e m L-C(D), \triangle A a p o B(E)$, and $\triangle A a p o B 48(F)$ The values are presented as mean $\pm S E M$. 
$\Delta \mathrm{TG}$ in the $\mathrm{H}$ trial was significantly increased compared with each fasting level at 1,2 , and $4 \mathrm{~h}(p<0.05)$ and returned to the fasting level at $6 \mathrm{~h}$. However, $\Delta \mathrm{TG}$ in the $\mathrm{CH}$ trial was significantly increased compared with each fasting level at $4 \mathrm{~h}$ and did not return to fasting level at $6 \mathrm{~h}(p<0.05$ for all). $\Delta \mathrm{TG}$ peaked at $2 \mathrm{~h}$ in the $\mathrm{H}$ trial and at $4 \mathrm{~h}$ in the $\mathrm{CH}$ trial. $\Delta \mathrm{TG}$ in the $\mathrm{C}$ trial showed no significant change during the $6 \mathrm{~h}$.

$\triangle \mathrm{hTRL}-\mathrm{TG}$ in the $\mathrm{H}$ trial was significantly increased compared with the fasting level at 1 and $2 \mathrm{~h}(p<0.05$ for both $)$ and returned to the fasting level at $4 \mathrm{~h} . \Delta \mathrm{hTRL}-\mathrm{TG}$ in the $\mathrm{CH}$ trial tended to be higher than the fasting level at $4 \mathrm{~h}(p=0.16)$ and $6 \mathrm{~h}(p=0.14)$. $\Delta \mathrm{hTRL}-\mathrm{TG}$ peaked at $2 \mathrm{~h}$ in the $\mathrm{H}$ trial and at $4 \mathrm{~h}$ in the $\mathrm{CH}$ trial. $\Delta \mathrm{hTRL}-\mathrm{TG}$ in the $\mathrm{C}$ trial showed no significant change during the $6 \mathrm{~h}$.

$\triangle \mathrm{RLP}-\mathrm{TG}$ in the $\mathrm{H}$ and $\mathrm{CH}$ trials was significantly increased at $1 \mathrm{~h}(p<0.05)$, it peaked at $2 \mathrm{~h}$ in the $\mathrm{H}$ trial and at $4 \mathrm{~h}$ in the $\mathrm{CH}$ trial, and in both trials did not return to the fasting values. $\triangle \mathrm{RLP}-\mathrm{TG}$ in the $\mathrm{C}$ trial showed no significant change during the $6 \mathrm{~h}$.

$\triangle$ RemL-C in the $\mathrm{H}$ trial was significantly higher than the fasting level at 2,4 , and $6 \mathrm{~h}$ ( $p<0.05$ for all). $\Delta$ RemL-C in the $\mathrm{CH}$ trial was significantly higher than the fasting level at 4 and $6 \mathrm{~h}$ ( $p<0.05$ for both). $\Delta$ RemL-C in the $\mathrm{H}$ and $\mathrm{CH}$ trials did not return to the fasting levels at the end of the experiment. The RemL-C peaked at $4 \mathrm{~h}$ in the $\mathrm{CH}$ trial, but plateaued from 1 to $6 \mathrm{~h}$ in the $\mathrm{H}$ trial. $\Delta$ RemL-C in the $\mathrm{C}$ trial showed no significant change during the $6 \mathrm{~h}$.

\subsection{Serum Concentrations of Fasting and Postprandial apoB and apoB 48}

The concentrations of fasting and postprandial apoB and apoB48 in the 3 trials are presented in Table 5, and time courses for $\triangle \mathrm{apoB}$ and $\triangle \mathrm{apoB} 48$ are shown in Fig. $1 \mathrm{E} \& \mathrm{~F}$.

$\triangle$ apoB was significantly decreased at $2 \mathrm{~h}$ in the $\mathrm{H}$ trial $(p<$ $0.05)$ and at 2 and $4 \mathrm{~h}$ in the $\mathrm{CH}$ trial $(p<0.05$ each). However, $\triangle$ apoB in the $\mathrm{C}$ trial did not decrease up to $4 \mathrm{~h}$ but significantly increased at $6 \mathrm{~h}(p<0.05)$.

$\triangle$ apoB48 in the $\mathrm{H}$ and $\mathrm{CH}$ trials was significantly increased at $1 \mathrm{~h}$ compared with each fasting value ( $p<0.05$ for all), and it peaked at $2 \mathrm{~h}$ in the $\mathrm{H}$ trial and at $4 \mathrm{~h}$ in the $\mathrm{CH}$ trial. $\triangle a p o B 48$ in the $\mathrm{H}$ and $\mathrm{CH}$ trials did not return to the fasting values at $6 \mathrm{~h} . \triangle \mathrm{apoB} 48$ in the $\mathrm{CH}$ trial was higher than that in the $\mathrm{H}$ trial at $4 \mathrm{~h}(p<0.05)$ and $6 \mathrm{~h}(p=0.09)$. The peak in the $\mathrm{CH}$ trial (at $4 \mathrm{~h}$ ) tended to be higher than that in the $\mathrm{H}$ trial (at 2 h) $(p=0.06) . \triangle \mathrm{AUC}$-apoB 48 in the $\mathrm{CH}$ trial $(11.3 \pm 1.0$ $\mu \mathrm{g} / \mathrm{h} / \mathrm{mL})$ tended to be larger than that in the $\mathrm{H}$ trial $(8.8 \pm 0.8$ $\mu \mathrm{g} / \mathrm{h} / \mathrm{mL}, p=0.07)$.

\section{Discussion}

The subjects examined in this study were normal and typical in physique and in basal carbohydrate and lipid profile as young healthy Japanese women. The basal conditions were not different among the 3 trials, and estimated to be appropriate for the study. Apo AI, AII, B(-100), B48, CII, CIII, and $\mathrm{E}$ measured in the present study are major components of lipoproteins. After the ingestion of a fatty meal, $\mathrm{CM}$ is assembled in enterocytes. CM contains dietary fat and apoB48 and $\mathrm{AI}$ as the major protein components. After acquiring apoCII and E, TG in CM is hydrolyzed by lipoprotein lipase (LPL), and removed. TG depletion results in a size reduction, referred to as CM remnant. This remnant is taken up by the liver [13]. Endogenous TG is put into VLDL and secreted by the liver, having apoB100, CII, CIII, and $\mathrm{E}$ as the main apolipoproteins [14]. After lipolysis by LPL, VLDL is metabolized to VLDL remnant and taken up by the liver, or finally converted to LDL. Therefore, we used various indices in order to estimate the postprandial course of exogenous and endogenous lipid and lipoprotein metabolism.

The major findings in this study are that the increase in postprandial apoB48 was delayed and its peak following the ingestion of an HFS-containing cola in combination with a hamburger was higher than that following the ingestion of a hamburger only in young healthy women. The peaks of the serum concentrations of TG, RLP-TG, RemL-C, and hTRL-TG after the ingestion of cola in combination with a hamburger were delayed compared with those after the ingestion of a hamburger only, demonstrating a delay in postprandial lipid metabolism. These changes of postprandial metabolism are consistent with our previous studies, indicating that this effect is due to the fructose contained in the cola $[8,9]$.

Lower insulin excursion and higher FFA concentration following the ingestion of fructose compared to glucose may also have resulted in the reduced activation of LPL $[8,9]$, a lipolytic enzyme on the capillary endothelium of extrahepatic tissues that catabolizes both exogenous and endogenous TRL-TG [15]. In the present study, although insulin was significantly increased in the $\mathrm{CH}$ trial, the apoB48 peak was delayed compared to that in the $\mathrm{H}$ trial, indicating delayed secretion or increased production of chylomicron by the small intestine. These results suggest that reduced activation of LPL may be less important. The delayed secretion of apoB48-containing lipoprotein particles may be explained by the difference between the absorption mechanisms of fructose and glucose in the small intestine, but we have no direct evidence for whether this explanation is correct or not. A study has examined intestinal and hepatic TRL particle production in response to the coinfusion of glucose or fructose with Intralipid into the duodenum [16]. Glucose stimulated TRL-apoB48 production concomitant with the increase in fractional clearance, thus net TRL-apoB48 increase was moderate; however, fructose enhanced TRL-apoB48 production without the increased clearance. In the present study, the peaks of TG and the indicators related to remnants, i.e., hTRL-TG, RLP-TG, RemL-C, and apoB48, were all delayed after the coingestion of hamburger and cola compared with those after the ingestion of hamburger only. Among these indicators, apoB48 is the direct index of exogenous lipoprotein number, and RLP-TG may be more sensitive to exogenous remnants, while the method for RemL-C may be suitable for endogenous remnants [13]. The present results 
showed that the metabolism of both exogenous and endogenous lipoproteins was delayed after the ingestion of hamburger and cola compared to the ingestion of hamburger only.

After the ingestion of a fructose-containing beverage without glucose or sucrose, serum lactate concentration is significantly increased $[8,9]$. In the present study, the serum lactate concentration rose markedly following the ingestion of cola and/or hamburger. Because the cola contained only $16.8 \mathrm{~g}$ fructose, $12.6 \mathrm{~g}$ glucose, and $8.1 \mathrm{~g}$ sucrose, this suggests that $350 \mathrm{~mL}$ of usual cola may be surplus for young Japanese women. When the glycolytic pathway is saturated by an excess influx of fructose, fructose may be converted to lactate [8] and fat by de novo lipogenesis, eventually enhancing VLDL-TG production [17].

It has been reported that sugar intake is associated with overweight or obesity among children in the USA and Europe $[18,19]$. However, in Japanese children, the average sugar intake from snacks and beverages is within the range of FAO/WHO recommendations [20]. In addition, sugar-sweetened beverage consumption in 18- to 20-year-old Japanese women (144 g/day) was much lower than that in 12to 19-year-old US youth (683 g/day) [21]. Subjects in Japan with higher sugar-sweetened beverage intake tend to consume less protein, dietary fiber, and most micronutrients than those with lower intake, and the consumption of sugar-sweetened beverages is related to the quality of diet [21]. Soft drink consumption, except for pure juice, was associated with an increased risk of type 2 diabetes in Japanese women [22]. Therefore, we recommend to not consume a large amount of an HFS-containing beverage concomitant with fatty food such as a hamburger even in young healthy women and to choose nonsugar beverages at meal time, such as Japanese tea, mineral water, and black coffee.

In the present study, we elucidated the adverse effect of single ingestion of an HFS-containing beverage (cola) and fatty fast food (hamburger), which young women often consume at the same time in daily life. The amounts ingested in this study are typical, not much for Japanese youth. We have been studying postprandial lipid metabolism using the Oral Fat Tolerance Test (OFTT) cream (Jomo, Takasaki, Japan; $1 \mathrm{~g} / \mathrm{kg}, 0.35 \mathrm{~g} / \mathrm{kg}$ as fat) $[8,9,23-26]$. In the present study, we showed that fatty food such as a hamburger has an effect similar to that of OFTT cream. A meta-analysis evaluated the diagnostic value of various fat tolerance tests and showed that a 4-h time point after oral fat loading is the most representative for measurement of TG [27]. The nonfasting TG levels may be useful for revealing early stages of dyslipidemia.

\section{Conclusion}

The ingestion of an HFS-containing beverage (cola) in combination with fatty food (a hamburger) delayed and extended both exogenous and endogenous postprandial lipid metabolism in healthy young women. Fructose contained in the cola probably delayed exogenous lipid metabolism. However, a limitation of the present study is the small number of subjects investigated, indicating that the obtained results should be considered with caution.

\section{Acknowledgements}

This work was supported by a Grant-in-Aid for Scientific Research (Basic Research C, JSPS KAKENHI Grant Number 24500874) from the Ministry of Education, Culture, Sports, Science and Technology of Japan. We thank Mr. Ryuji Hayashi for his technical assistance. We also thank Ms. Eri Ikeda, Ms. Chiharu Iijima, and Ms. Aiko Tanaka for their technical cooperation.

\section{References}

[1] Ministry of Agriculture, Forestry and Fisheries, Japan, 2014. http://www.maff.go.jp/j/seisan/tokusan/kansho/pdf/26sy-1.pdf (in Japanese)

[2] Walker RW, Dumke KA, Goran MI: Fructose content in popular beverages made with and without high-fructose corn syrup. Nutrition, 2014; 30: 928-935

[3] Te Morenga L, Mallard S, Mann J: Dietary sugars and body weight: systematic review and meta-analyses of randomised controlled trials and cohort studies. BMJ, 2012; 346: e7492

[4] Malik VS, Pan A, Willett WC, Hu FB: Sugar-sweetened beverages and weight gain in children and adults: a systematic review and meta-analysis. Am J Clin Nutr, 2013; 98: 1084-1102

[5] Eshak ES, Iso H, Kokubo Y, Saito I, Yamagishi K, Inoue M, Tsugane S: Soft drink intake in relation to incident ischemic heart disease, stroke, and stroke subtypes in Japanese men and women: the Japan Public Health Centre-based study cohort I. Am J Clin Nutr, 2012; 96: 1390-1397

[6] Stanhope KL, Bremer AA, Medici V, Nakajima K, Ito Y, Nakano T, Chen G, Fong TH, Lee V, Menorca RI, Keim NL, Havel PJ: Consumption of fructose and high fructose corn syrup increase postprandial triglycerides, LDL-cholesterol, and apolipoprotein-B in young men and women. $\mathrm{J}$ Clin Endocrinol Metab, 2011; 96: 1596-1605

[7] Zilversmit DB: Atherogenesis: a postprandial phenomenon. Circulation, 1979; 60: 473-485

[8] Saito H, Kagaya M, Suzuki M, Yoshida A, Naito M: Simultaneous ingestion of fructose with fat exacerbates postprandial exogenous lipidemia in young healthy Japanese women. J Atheroscler Thromb, 2013; 20: 591-600

[9] Saito H, Kato M, Yoshida A, Naito M: The ingestion of a fructose-containing beverage combined with fat cream exacerbates postprandial lipidemia in young healthy women. J Atheroscler Thromb, 2015; 22: 85-94

[10] Asano M, Fukakura N, Odachi J, Kawaraya C, Nanba A, Yasuda N, Yamamoto E: Use of Fast Foods among Young People. J Nutr Diet, 2003; 61: 47-54

[11] Matthews DR, Hosker JP, Rudenski AS, Naylor BA, Treacher DF, Turner RC: Homeostasis model assessment: insulin resistance and beta-cell function from fasting plasma glucose and insulin concentrations in man. Diabetologia, 1985; 28: 412-419 
[12] Okada M, Saito T, Yoshimura H, Noguchi Y, Ito T, Sasaki H, Hama H. Surfactant-based homogeneous assay for the measurement of triglyceride concentrations in VLDL and intermediate-density lipoprotein. Clin Chem, 2005; 51: $1804-1810$

[13] Sato I, Ishikawa Y, Ishimoto A, Katsura S, Toyokawa A, Hayashi F, Kawano S, Fujioka Y, Yamashita S, Kumagai S: Significance of measuring serum concentrations of remnant lipoproteins and apolipoprotein B-48 in fasting period. $\mathrm{J}$ Atheroscler Thromb, 2009; 16: 12-20

[14] Gabor J, Spain M, Kalant N: Composition of serum very-low-density and high-density lipoproteins in diabetes. Clin Chem, 1980; 26: 1261-1265

[15] Brunzell JD, Hazzard WR, Porte D Jr, Bierman EL: Evidence for a common, saturable, triglyceride removal mechanism for chylomicrons and very low density lipoproteins in man. J Clin Invest, 1973; 52: 1578-1585

[16] Xiao C, Dash S, Morgantini C, Lewis GF: Novel role of enteral monosaccharides in intestinal lipoprotein production in healthy humans. Arterioscler Thromb Vasc Biol, 2013; 33: 1056-1062

[17] Hudgins LC, Parker TS, Levine DM, Hellerstein MK: A dual sugar challenge test for lipogenic sensitivity to dietary fructose. J Clin Endocrinol Metab, 2011; 96: 861-868

[18] Poti JM, Slining MM, Popkin BM: Solid fat and added sugar intake among U.S. children: The role of stores, schools, and fast food, 1994-2010. Am J Prev Med, 2013; 45: 551-559

[19] Aeberli I, Zimmermann MB, Molinari L, Lehmann R, l'Allemand D, Spinas GA, Berneis K: Fructose intake is a predictor of LDL particle size in overweight schoolchildren. Am J Clin Nutr, 2007; 86: 1174-1178
[20] Takeichi H, Taniguchi H, Fukinbara M, Tanaka N, Shikanai S, Sarukura N, Hsu TF, Wong Y, Yamamoto S: Sugar intakes from snacks and beverages in Japanese children. J Nutr Sci Vitaminol, 2012; 58: 113-117

[21] Yamada M, Murakami K, Sasaki S, Takahashi Y, Okubo H: Soft drink intake is associated with diet quality even among young Japanese women with low soft drink intake. J Am Diet Assoc, 2008; 108: 1997-2004

[22] Eshak ES, Iso H, Mizoue T, Inoue M, Noda M, Tsugane S: Soft drink, $100 \%$ fruit juice, and vegetable juice intakes and risk of diabetes mellitus. Clin Nutr, 2013; 32: 300-308

[23] Nabeno Y, Fukuchi Y, Matsutani Y, Naito M: Influence of aging and menopause on postprandial lipoprotein responses in healthy adult women. J Atheroscler Thromb, 2007; 14: $142-150$

[24] Nabeno-Kaeriyama Y, Fukuchi Y, Hayashi S, Kimura T, Tanaka A, Naito M: Delayed postprandial metabolism of triglyceride-rich lipoproteins in obese young men compared to lean young men. Clin Chim Acta, 2010; 411: 1694-1699

[25] Hashimoto S, Ootani K, Hayashi S, Naito M: Acute effects of shortly pre-versus postprandial aerobic exercise on postprandial lipoprotein metabolism in healthy but sedentary young women. J Atheroscler Thromb, 2011; 18: 891-900

[26] Hashimoto S, Hayashi S, Yoshida A, Naito M: Acute effects of postprandial aerobic exercise on glucose and lipoprotein metabolism. J Atheroscler Thromb, 2013; 20: 204-213

[27] Mihas C, Kolovou GD, Mikhailidis DP, Kovar J, Lairon D, Nordestgaard BG, Ooi TC, Perez-Martinez P, Bilianou H, Anagnostopoulou K, Panotopoulos G: Diagnostic value of postprandial triglyceride testing in healthy subjects: a meta-analysis. Curr Vasc Pharmacol, 2011; 9: 271-280 